\title{
Endovascular Treatment of Proximal Superior Mesenteric Artery Pseudoaneurysm after Stab Injury
}

\author{
Dong Hun Kim, M.D. ${ }^{1}$, Young-Wook Kim, M.D., Ph.D. ${ }^{2}$, and Kwang Bo Park, M.D., Ph.D. ${ }^{3}$ \\ 1 Department of Surgery, Dankook University Hospital, Cheonan; 'Division of Vascular Surgery, Department of Surgery, ${ }^{3}$ Department of Radiology, Samsung Medical Center, \\ Sungkyunkwan University School of Medicine, Seoul, Korea
}

Superior mesenteric artery (SMA) injuries remain a challenge to most trauma surgeons and continue to result in significant mortality despite aggressive management. We report successful management of a proximal SMA injury through endovascular treatment in a 56-year-old man. The patient presented with hypotension due to a stab wound after the epigastrium. He underwent emergency laparotomy and repair of the penetrated wall of the stomach. Right retroperitoneal hematoma at the initial laparotomy expanded and ruptured, requiring a massive transfusion. At the second laparotomy, we performed lateral arteriorrhaphy of the proximal SMA. Follow-up abdominal computed tomography angiography on post-injury day 7 showed a pseudoaneurysm on the left side of the SMA trunk. The patient was treated successfully with an endovascular stent graft.

Key Words: endovascular procedure; superior mesenteric artery; trauma.

Superior mesenteric artery (SMA) injuries are uncommon and lethal injuries that are infrequently treated. This type of injury poses a unique challenge because of the risk of massive hemorrhage, associated injuries to the pancreas, mesenteric vein or aorta, and bowel ischemia. An incidence of $0.09 \%$ was reported with high mortality, mostly due to penetrating trauma in young males.[1] Immediate surgical laparotomy is the treatment of choice in hemodynamically instable patients. Over the past several decades, endovascular treatment has been used with increasing frequency to manage vascular trauma. Endovascular treatment of SMA pseudoaneurysm can lead to technical success and is a feasible treatment option in hemodynamically stable patients.[2] Herein we report successful endovascular treatment of a pseudoaneurysm caused by a stab injury to the SMA.

\section{Case Report}

A 56-year-old man was admitted to the emergency room with a stab injury at the mid-epigastrium. On arrival, he was alert with a pulse rate of 109 beats/min, blood pressure of 83/47

Received on August 5, 2015 Revised oon September 25, 2015 Accepted on September 25, 2015

Correspondence to: Young-Wook Kim, Division of Vascular Surgery, Department of Surgery, Samsung Medical Center, Sungkyunkwan University School of

Medicine, 81 Irwon-ro, Gangnam-gu, Seoul 06351, Korea

Tel: +82-2-3410-3461, Fax: +82-2-3410-0040

E-mail: ywkim@skku.edu

${ }^{*}$ No potential conflict of interest relevant to this article was reported.
$\mathrm{mmHg}$, respiratory rate of $28 / \mathrm{min}$, and hemoglobin level of $10.6 \mathrm{~g} / \mathrm{dL}$. Physical examination revealed tenderness on the upper abdomen and abdominal distension. Focused assessment with sonography for trauma (FAST) showed intraabdominal fluid in the Morison pouch and splenorenal recess. After the patient was resuscitated with $1,500 \mathrm{~mL}$ intravenous crystalloid fluids and 2 units of packed red cells, his hemody- 
namics improved with a blood pressure of 107/63 mmHg. Abdomen computed tomography (CT) showed perigastric free air with intragastric hematoma, fluid collection in the perihepatic and perisplenic spaces, and right retroperitoneal hematoma in the front of the right kidney without contrast extravasation. The patient was transferred to the operating room. Exploration of the abdominal cavity revealed about $600 \mathrm{~mL}$ of blood in the peritoneal cavity, a thin laceration of the left lobe of the liver, and penetration of the anterior and posterior wall of the distal antrum of the stomach. There was a $7 \times 5 \mathrm{~cm}$ hematoma at the mesenteric root around the pancreas neck without pancreas injury. When we accessed the superior mesenteric vessels below inferior pancreas margin through the greater omental sac, we were not able to find an active bleeding focus at the mesenteric root. Gastric injuries were primarily repaired with a stapler. A nonexpanding hematoma at the right retroperitoneal hematoma was not entered, and hemostatic agent $\left(\right.$ TachoSil ${ }^{\circledR}$; Takeda $\mathrm{GmbH}$, Linz, Austria) was used around the superior mesenteric vessels below inferior pancreas margin, because of the venous oozing for dissection.

At post-injury day (PID) 1, there were signs of ongoing bleeding with over 3,500 mL blood collected via a JacksonPratt drain placed at the right retroperitoneal area. The

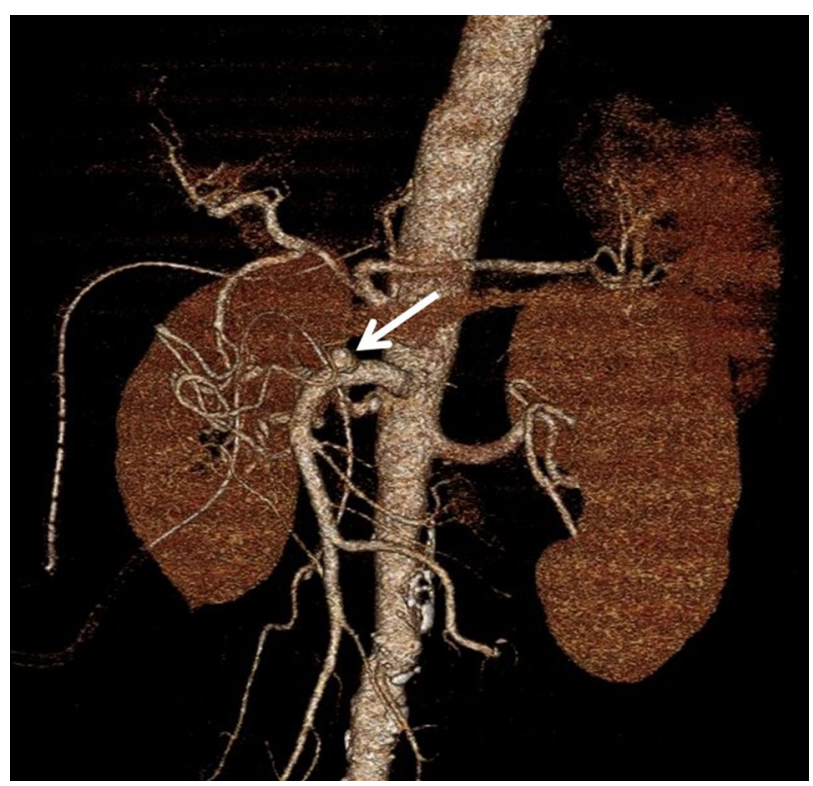

Fig. 1. Abdominal computed tomography angiography on postinjury day 7 showed an unruptured pseudoaneurysm (white arrow) of the anterior wall of the superior mesenteric artery trunk just proximal to the inferior pancreaticodudoneal artery. patient received 10 units of packed red blood cells, 8 units of fresh frozen plasma, and 10 units of platelet concentrate. His hemodynamics were preserved with intravenous use of a vasopressor agent. He had severe abdominal pain and back pain. The abdomen was moderately distended and diffusely tender. Abdominal CT showed a huge hematoma at the right retroperitoneum with extravasation of the contrast agent from the right side of the proximal SMA. Selective SMA angiography did not reveal a definite bleeding site. Thus, we decided to perform an immediate reoperation. Intraoperatively, about $2,500 \mathrm{~mL}$ of free blood and clots were evacuated from the right retroperioneal space. Proximal SMA was approached with right-sided medial visceral rotation mobilizing the pancreas head and duodenum cephalad and medially, and the root of the SMA was exposed. We found a longitudinal laceration at the right side of the root of the SMA with active bleeding. Lateral arteriorrhaphy was performed using 4-0 prolene interrupting sutures to preserve SMA patency. The patient thereafter had an uneventful recovery.

On follow-up abdomen CT angiography on PID 7, we found a $1-\mathrm{cm}$ pseudoaneurysm at the anterior wall of the SMA trunk proximal to the inferior pancreaticodudoneal

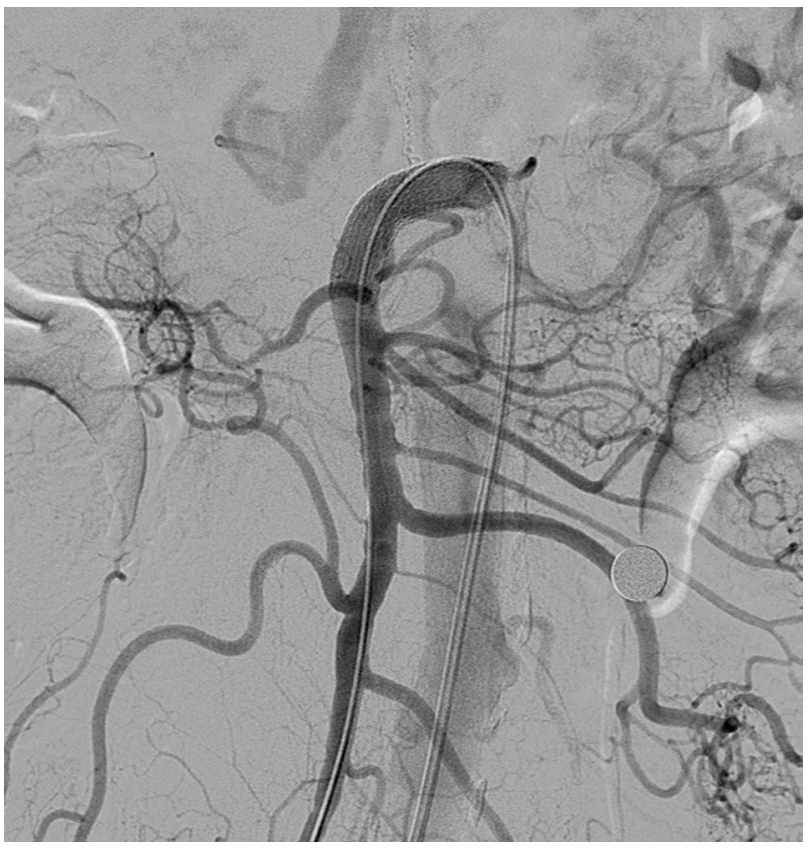

Fig. 2. Superior mesenteric artery (SMA) angiogram revealed a covered stent placed in the proximal SMA covering the pseudoaneurysm. 


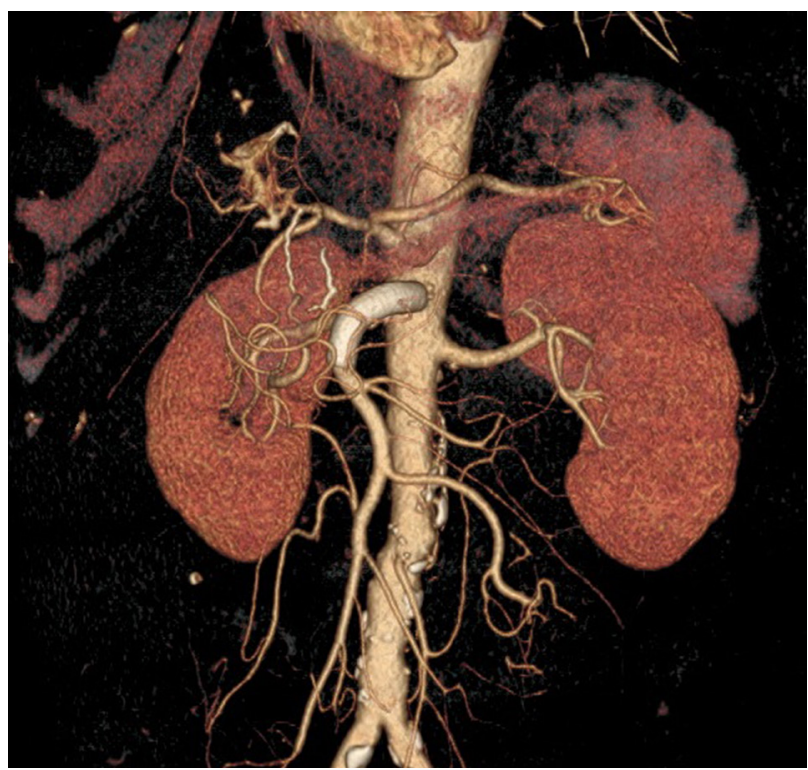

Fig. 3. Follow-up computed tomography angiography one month after percutaneous endovascular treatment showed the patentcovered stent of the superior mesenteric artery and the excluded pseudoaneurysm.

artery (Fullen's anatomic zone 1), which could be near the repair site of the proximal SMA (Fig. 1). After treatment of paralytic ileus for 20 days, the patient was transferred to a tertiary referral center for treatment of the SMA pseudoaneurysm. SMA was accessed at the right femoral artery and a 9-Fr guiding sheath tip was positioned at the SMA orifice. A covered stent (Viabahn ${ }^{\circledR}$; W.L. Gore \& Associates, Flagstaff, Ariz; diameter, $9 \mathrm{~mm}$; length, $5 \mathrm{~cm}$ ) was successfully implanted in the SMA covering the pseudoaneurysm (Fig. 2). On follow-up abdomen CT angiography 1 month after stent graft insertion, the covered stent of the SMA was patent and the pseudoaneurysm was successfully excluded (Fig. 3 ). The patient was discharged with prescription of the antiplatelet agent aspirin and clopidogrel to prevent thrombotic occlusion of the stent graft without further complications.

\section{Discussion}

SMA injuries are quite rare, although their true incidence is unknown, they comprise significantly less than $1 \%$ of all admissions to trauma centers.[1] SMA injury carries a high risk of morbidity and mortality. Overall mortality rates range from $33 \%$ to $75 \%$.[1,3] Early death is a result of ex- sanguination, whereas late death stems from multisystem organ failure and sepsis. Fullen et al.[4] proposed a classification scheme based on the extent of ischemia and anatomic zones of injury, recognizing the unique technical challenges posed by injuries in different zones. Fullen's anatomic zones correlated with mortality. A multiinstitutional study showed that SMA trunk injury proximal to the inferior pancreaticoduodenal artery, Fullen's anatomic zone 1, was associated with the highest mortality rate of $76.5 \%$.[3] In this case, SMA injury belonged to this zone.

A stab injury to the SMA might be missed in initial diagnosis, even in emergency laparotomy. In our case, contrast extravasation near the SMA was not detected on abdominal $\mathrm{CT}$, and there was a small hematoma in the right retroperitoneum, which we found to be a non-expanding hematoma during the initial operation. There are few reports of delayed SMA rupture. Reynard et al.[5] reported presentation of a SMA rupture 5 days after the initial blunt injury. Asensio et al.[3] reported that $96 \%$ of operative finding in SMA injuries had retroperitoneal hematomas, especially $73 \%$ in Fullen's anatomic zone 1. Because a penetrating injury of the proximal SMA can be overlooked by routine laparotomy, we should realize that the retroperitoneal hematoma even a small or non-expanding could be a definitive sign of the proximal SMA injury.

Depending on the extent of arterial injury, several methods of arterial reconstruction are available including primary suture, ligation, and autogenous or synthetic graft interposition, and should be used selectively according to the injury zone.[6] A proximal SMA injury, Fullen's anatomic zone 1 , should be repaired either primarily or with an interposition graft, because of viability of midgut.[3] In a case with limited injury involving a portion of the artery wall, lateral arteriorrhaphy could be possible. In this case, lateral arteriorrhaphy to the proximal SMA injury was successfully performed with right-sided medial visceral rotation, CattellBraasch maneuver,[7] because the abdominal CT revealed contrast extravasation in the right side wall of the SMA trunk with a huge hematoma at the right retroperitoneum. Endovascular approach could be another treatment option in the patient with stable hemodynamics. However, operation was a treatment choice in this case, because extravasation of the proximal SMA in the selective SMA angiography was not found and hemodynamics of our patient was aggravated 
in hemorrhagic shock.

Our case showed unusually late presentation of the unruptured pseudoaneurysm in proximal SMA wall that previously might be a part of the repair site, which was confirmed on follow-up CT 6 days after the second operation. Technical failure of surgical repair would be likely to present with a pseudoaneurysm, because abdomen CT on PID 1 did not reveal except extravasation from the right side of the proximal SMA. A multiinstitutional study reported that $1 \%$ of complications after surgical procedure were pseudoaneurysm.[3]

Treatment of SMA pseudoaneurysms is warranted even in asymptomatic patients because of the risk of thrombosis or rupture with resultant acute mesenteric ischemia and death. [2] Conventional treatment of SMA pseudoaneurysms involves open surgical ligation or aneurysm resection with an interposition graft. However, these injuries pose a surgical challenge that tests the skills of even the most experienced trauma surgeon. With endovascular treatment, extensive surgical dissection, risk of bleeding, and surrounding organ damage can be avoided. Since the first report of covered stent placement in 1998,[8] over 30 cases of successful covered stent placements have been reported.[2] Endovascular treatment is recommended, especially in hemodynamically stable patients, although few studies have compared outcomes between surgical and endovascular treatment of SMA pseudoaneurysms. We felt that embolization was inappropriate in our case due to the size and position of the pseudoaneurysm. Therefore, we elected to use a selfexpanding Viabahn covered stent, which is a nitinol metal stent covered with polytetrafluoroethylene, to exclude the pseudoaneurysm. Follow-up CT angiography 1-3 months after covered stent placement is recommended for surveillance of endoleak and stent patency.[2] Our patient had no subsequent episodes of bleeding or bowel ischemia, and follow-up CT performed 1 month after stent placement confirmed stent patency, which we ascribed to antiplatelet therapy after covered stent placement.

In summary, small retroperitoneal hematoma should not be overlooked for proximal SMA injury. Percutaneous endovascular treatment using a covered stent for the treatment of SMA pseudoaneurysm, after surgical repair of the proximal SMA injury, could lead to technical success and may be a feasible treatment option. Furthermore, angiography (either CT angiography or percutaneous angiography) should be performed to detect pseudoaneurysm, after arterial repair due to trauma.

\section{References}

1) Asensio JA, Berne JD, Chahwan S, Hanpeter D, Demetriades D, Marengo J, et al: Traumatic injury to the superior mesenteric artery. Am J Surg 1999; 178: 235-9.

2) Kim SK, Lee J, Duncan JR, Picus DD, Darcy MD, Sauk S: Endovascular treatment of superior mesenteric artery pseudoaneurysms using covered stents in six patients. AJR Am J Roentgenol 2014; 203: 432-8.

3) Asensio JA, Britt LD, Borzotta A, Peitzman A, Miller FB, Mackersie RC, et al: Multiinstitutional experience with the management of superior mesenteric artery injuries. J Am Coll Surg 2001; 193: 354-65; discussion 365-6.

4) Fullen WD, Hunt J, Altemeier WA: The clinical spectrum of penetrating injury to the superior mesenteric arterial circulation. J Trauma 1972; 12: 656-64.

5) Reynard J, Singh A, Tiwari A, Peters JL: Delayed presentation of superior mesenteric artery rupture following blunt trauma. Injury 2004; 35: 1306-7.

6) Weinstein D, Wiener Y, Bass A, Halevy A: Traumatic laceration of the superior mesenteric artery: report of a case and review of the literature. J Trauma 2002; 53: 568-70.

7) Cattell RB, Braasch JW: A technique for the exposure of the third and fourth portions of the duodenum. Surg Gynecol Obstet 1960; 111: 378-9.

8) McGraw JK, Patzik SB, Gale SS, Dodd JT, Boorstein JM: Autogenous vein-covered stent for the endovascular management of a superior mesenteric artery pseudoaneurysm. J Vasc Interv Radiol 1998; 9: 779-82. 